\title{
Efficacy of Mometasone Nasal Spray in Children with Snoring due to Adenoids
}

\author{
${ }^{1}$ Vipan Gupta, ${ }^{2}$ Monika Gupta, ${ }^{3}$ Prithpal S Matreja, ${ }^{4}$ Sunder Singh
}

\begin{abstract}
Background: Snoring and OSA is very commonly seen among the pediatric population, most commonly due to adenoid and tonsil hypertrophy which requires adenotonsillectomy. The effect of this surgery on the immunity is controversial. This study was planned to see any alternative therapy which can delay or avoid the surgery by providing symptomatic relief to the patient.
\end{abstract}

Objective: To see the effect of intranasal mometasone on nasal obstruction due to adenoids, hence relieving the OSA and snoring.

Design: Prospective and observational study.

Materials and methods: Fifty-five children having snoring and or OSA due to adenoids were given intranasal mometasone. The symptoms before and after treatment were compared using OSA 18 questionnaire.

Results: There was significant improvement in all the domains of OSA 18. The average total score showed improvement from 56.33 to 51.51 which is significant $(p<0.001)$.

Conclusion: The use of intranasal steroids is easy and effective method to improve nasal obstruction, snoring and OSA among children having adenoid hypertrophy.

Keywords: Obstructive sleep apnea syndrome, Intranasal steroids, Adenoid hypertrophy.

How to cite this article: G upta V, G upta M, Matreja PS, S ingh $S$. Efficacy of Mometasone Nasal S pray in Children with S noring due to Adenoids. Clin R hinol An Int J 2014;7(1):1-4.

\section{Source of support: Nil}

Conflict of interest: None

\section{INTRODUCTION}

A denoid hypertrophy is known to be associated with several harmful clinical conditions ${ }^{1,2}$ which range from mild nasal

\footnotetext{
${ }^{1,3}$ Associate Professor, ${ }^{2}$ Assistant Professor, ${ }^{4} \mathrm{P}$ rofessor and Head

${ }^{1,4}$ Department of ENT, Gian Sagar Medical College and Hospital, Patiala, Punjab, India

${ }^{2}$ Department of Obstetrics and Gynecology, Gian Sagar Medical College and Hospital, Patiala, Punjab, India

${ }^{3}$ Department of Pharmacology, Gian Sagar Medical College and Hospital, Patiala, Punjab, India
}

Corresponding Author: Vipan Gupta, Associate Professor Department of ENT, 45 Preet Nagar Lower Mall, Patiala Punjab-147001, India, e-mail: drvipan@yahoo.com obstruction, otitis media to severe obstructive sleep apnea syndrome (OSA S). Delayed diagnosis and treatment of these diseases may result in consequences such as behavior alterations, low growth and weight gain, craniofacial alterations secondary to mouth breathing, mastication and swallowing disorders, in addition to cor pulmonale and left heart failure. ${ }^{3}$ OSA S in children is usually managed with adenotonsillectomy which needs general anesthesia and sometimes is associated with complications. A s adjunctive treatments, medical methods for reduction of adenoid size are limited. However, recent studies showed that topical nasal corticosteroid spray reduced adenoid size and improved symptoms of nasal airway obstruction and OSA S. ${ }^{4}$ The clinical data in Indian population is still limited hence this present study is planned to see the clinical efficacy of mometasone on patients with adenoid hypertrophy.

\section{MATERIALS AND METHODS}

This prospective, observational study was approved by the Institutional Ethics Committee of Gian Sagar M edical College and Hospital, Patiala. Fifty-five patients (31 males, 24 females), ranging from 4 to 12 -year-old, who visited Otolaryngology Department due to snoring and nasal obstruction were selected between M arch 2013 to October 2013. The following patient were included (1) history of habitual snoring for the last 3 months or longer, and (2) adenoid hypertrophy confirmed with simple X-ray findings or endoscopic examination by otolaryngologist. Children were excluded from the study if they met any of the following exclusion criteria: (1) presence of symptoms of acute respiratory infection; (2) use of nasal or systemic corticosteroid or antibiotics within 4 weeks prior to the study; (3) prior tonsil or adenoid surgery; and (4) a history of craniofacial, neuromuscular or genetic disorders.

Informed consent for participation in this study was obtained from the parent or legal guardian of each child enrolled. Initial assessment of each patient upon entering the study included the following: history and physical examination (including body weight and height), parental Obstructive Sleep A pnea questionnaire (OSA-18, Table 1) and adenoid $X$-ray. Patients were diagnosed to have allergic rhinitis if showing typical clinical symptoms and raised eosinophil count. Body mass index was calculated and more 
than 25 were considered as obese. A fter the enrolment, the children were put on the intranasal mometasone therapy. The outcome of intranasal mometasone was done using the same OSA 18 questionnaire after the period of 4 weeks.

The OSA-18, is a valid and reliable discriminative quality of life $(\mathrm{Q} O L)$ measure for children with varying levels of sleep disabled breathing. ${ }^{5}$ The survey consists of 18 items grouped into 5 domains: sleep disturbance (4 items), physical suffering (4 items), emotional distress ( 3 items), daytime problems ( 3 items), and caregiver concerns (4 items). Items are scored on a 7-point ordinal scale and have excellent test-retest reliability.

\section{STATISTICAL ANALYSIS}

All the data assembled was presented as mean \pm standard deviation. Results were analyzed with the help of appropriate parametric (two tailed student's t-test) test. A $p<0.05$ was considered as statistically significant.

\section{RESULTS}

A ll the 55 patients enrolled in the study completed the study. Table 1 presents the general information of all the 55 patients who took part in the study. Intranasal mometasone was given in 55 patients for 4 weeks. A ll the patients completed the trail with minimal side effects like dry mouth and epistaxis. Three patients reported minimal epistaxis, which got controlled without any medical aid. Parents of 3 patients who w ere less than 6 years of age had some difficulty using nasal steroids as their child was not cooperative.

Table 2 shows the changes of OSA 18 survey score before and after treatment. The average total score showed improvement from 56.33 to 51.51 which is significant $(p<0.001)$. There is significant improvement in all the five domins of OSA 18 score (Graph 1). A II the parents reported that there was significant improvement in the sleep and snoring, and is evident by reduction in scores 3.29 to 2.93 and 3.11 to 2.93 respectively. With the treatment of intranasal mometasone the problem of rhinorrhea also improved markedly $(p<0.001)$ with average score of 2.91 .

A mong the 55 patients 21 patients showed allergic features, with the intranasal therapy the allergic symptoms also subsided but the allergy had no affect on the efficacy of intranasal mometasone. Ten patients were found to have obesity. However, there was no change in body mass index

Table 1: Demographic features of patients

\begin{tabular}{ll}
\hline Features & Number \\
\hline No. of patients & 55 \\
Mean age (years) & 7.3 \\
Sex & 31 male, 24 female \\
Allergic features & 21 \\
Obesity & 10 \\
\hline
\end{tabular}

after the treatment and there was significant improvement in both obese and nonobese patients.

\section{DISCUSSION}

The palatine and pharyngeal tonsils, along with the lingual and tubal tonsils and the lateral pharyngeal bands are the important structures of Waldeyer's ring. They are secondary lymphoid organs, part of the mucosa-associated lymphoid tissue (MALT), which present immune activity mainly between 4 and 10 years of age. ${ }^{6}$ A denoidal hypertrophy is most common cause of obstructive sleep apnea and the cardiopulmonary syndrome, as severe complication. A denoidal hypertrophy is one of the mostfrequentindications for surgery in childhood, and adenoidectomy commonly is considered definitive treatment for nasopharyngeal obstruction. ${ }^{7}$ A lthough adenotonsillectomy is the most commonly performed procedure in children, its true impact upon the pediatric immune system is controversial. ${ }^{8}$ Encouraging results concerning chronic obstructive nasal symptoms due to adenoid in pediatric population were reported by use of intranasal steroid for short period. In order to see any alternative modality which can prevent or delay the requirement of adenoidectomy, we evaluated the utility of mometasone furoate aqueous nasal spray in children with adenoidal hypertrophy.

Chadha and Zhang ${ }^{9}$ reviewed seven studies including a total of 493 patients and found six of these studies demonstrated significant efficacy of various nasal steroids (mometasone, beclomethasone, flunisolide) in improving nasal obstruction symptoms and in reducing adenoid size, as measured with symptom scores and fiber-optic nasopharyngeal endoscopy, respectively. The response appeared to be a group effect and may be maintained longerterm by continuing nasal steroids at a lower maintenance

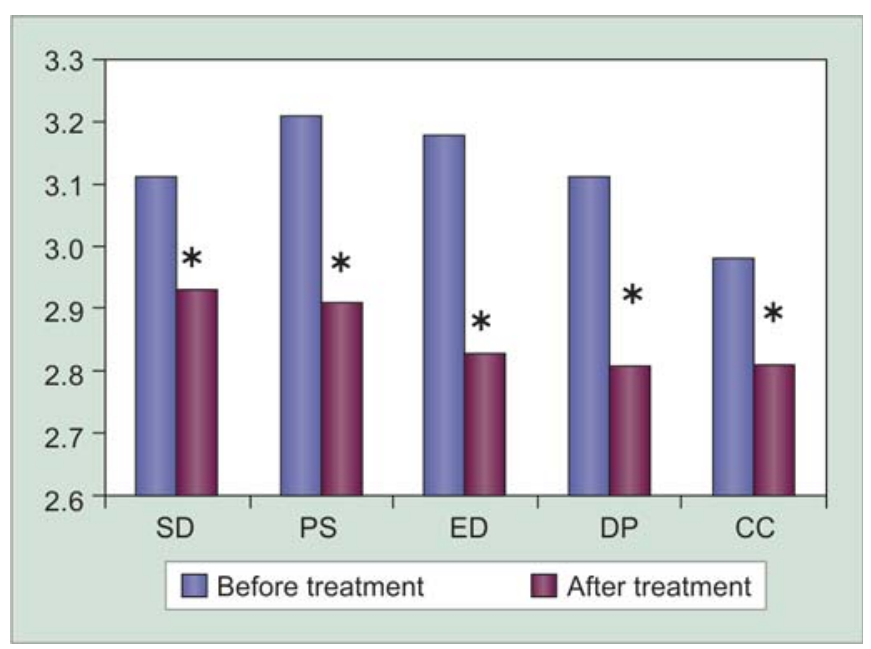

Graph 1: Comparison of symptom score between pre and post-4 weeks course of topical steroid treatment (SD: Sleep disturbance; PS: Physical symptoms; ED: Emotional distress; DP: Daytime problems; CC: Caregiver concerns); ${ }^{*} p<0.05$ 
Table 2: Parental questionnaire (OSA-18) results before and after intranasal corticosteroids treatment

\begin{tabular}{|c|c|c|c|}
\hline & Before treatment & After treatment & $\mathrm{p}$-value \\
\hline Sleep disturbance & $12.69 \pm 1.88$ & $11.73 \pm 0.97$ & $<0.001$ \\
\hline - Loud snoring & $3.11 \pm 0.50$ & $2.93 \pm 0.26$ & $<0.001$ \\
\hline - Breath holding/pause & $3.16 \pm 0.50$ & $2.95 \pm 0.23$ & $<0.001$ \\
\hline - Choking or gasping & $3.13 \pm 0.51$ & $2.93 \pm 0.26$ & 0.001 \\
\hline - Fragmented sleep & $3.29 \pm 0.66$ & $2.93 \pm 0.33$ & $<0.001$ \\
\hline Physical symptoms & $12.84 \pm 2.16$ & $11.64 \pm 1.25$ & $<0.001$ \\
\hline - Mouth breathing & $3.24 \pm 0.69$ & $2.93 \pm 0.38$ & $<0.001$ \\
\hline - Frequent URI & $3.29 \pm 0.71$ & $2.96 \pm 0.38$ & $<0.001$ \\
\hline - Rhinorrhea & $3.20 \pm 0.78$ & $2.91 \pm 0.48$ & $<0.001$ \\
\hline - Dysphagia & $3.11 \pm 0.76$ & $2.84 \pm 0.46$ & $<0.001$ \\
\hline Emotional distress & $9.55 \pm 2.14$ & $8.49 \pm 1.29$ & $<0.001$ \\
\hline - Mood swings or tantrums & $3.25 \pm 0.87$ & $2.87 \pm 0.51$ & $<0.001$ \\
\hline - Aggression/hyperactive & $3.11 \pm 0.90$ & $2.80 \pm 0.56$ & $<0.001$ \\
\hline - Discipline problems & $3.18 \pm 0.82$ & $2.82 \pm 0.55$ & $<0.001$ \\
\hline Daytime functions & $9.33 \pm 2.19$ & $8.42 \pm 1.40$ & $<0.001$ \\
\hline - Daytime drowsiness & $3.13 \pm 0.82$ & $2.82 \pm 0.51$ & $<0.001$ \\
\hline - Poor attention span & $3.18 \pm 0.93$ & $2.82 \pm 0.58$ & $<0.001$ \\
\hline - Difficulty awaking & $3.02 \pm 0.85$ & $2.78 \pm 0.57$ & $<0.001$ \\
\hline Caregiver concerns & $11.93 \pm 1.77$ & $11.24 \pm 1.12$ & $<0.001$ \\
\hline - Worried over child health & $3.00 \pm 0.75$ & $2.80 \pm 0.49$ & 0.001 \\
\hline - Concerned not enough air & $3.02 \pm 0.71$ & $2.85 \pm 0.49$ & 0.002 \\
\hline - Missed activities & $3.00 \pm 0.69$ & $2.84 \pm 0.46$ & 0.002 \\
\hline - Frustration & $2.91 \pm 0.70$ & $2.75 \pm 0.48$ & 0.002 \\
\hline Total & $56.33 \pm 5.29$ & $51.51 \pm 3.29$ & $<0.001$ \\
\hline
\end{tabular}

URI: Upper respiratory infection; OSA-18: Obstructive sleep apnea-18; $p<0.05$ : Statistically significant using paired student 't' test

dose. The treatment was safe and well-tolerated with few minor adverse events. In the present study we used the mometasone nasal spray as the data on Indian population is limited. We used OSA 18 questionnaire in order to evaluate the effects of mometasone which is a standard protocol for quality of life evaluation in cases of obstructive sleep apnea due to adenoids.

B erlucchi and Valetti ${ }^{10}$ eval uated theutility of mometasone furoate aqueous nasal spray in children with adenoidal hypertrophy in long-term maintenance therapy. Their study describes the long-term follow-up of children undergoing mometasone furoate aqueous nasal spray treatment for adenoidal hypertrophy. Voluntary suspension of maintenance therapy favors surgery of this disorder, whereas its regular administration may lead to successful results. In the present study short term use of intranasal mometasone has shown to decrease the adenoid size and symptom improvement. Rezende ${ }^{11}$ used the mometasone with saline irrigation and found the combination useful, but in the present study the intranasal mometasone alone was found to be sufficient for improvement. Intranasal mometasone has no effect on the tonsillar hypertrophy in the present study. Several mechanisms, such as direct lymphocytic action, inhibition of inflammation, and alteration of adenoid bacterial flora, have been suggested to explain how steroids decrease adenoid pad volume and improve symptoms of adenoidal hypertrophy, although none has yet achieved widespread acceptance. ${ }^{12}$
A kker ${ }^{13}$ et al analyzed 123 adenotonsillectomy patients for 12 months and reported significant decreased in IgA levels, in 1996, M ira ${ }^{7}$ et al followed 30 Brazilian patients aged between 3 and 15 years for 1 to 12 months. M inor reductions were reported on immunoglobulin levels. There is no data available on the affect of intranasal steroids on the immune system.

\section{CONCLUSION}

The available evidence suggests that short term use of nasal steroids may significantly improve nasal obstruction symptoms in children with adenoid hypertrophy. This improvement appears to be associated with a reduction of adenoid size. Evidence of long-term efficacy is awaited. Further studies are required to support the use of nasal steroids as a first-line approach in these children.

\section{REFERENCES}

1. Pagella F, Colombo A, Gatti O, Giourgos G, Matti E. Rhinosinusitis and otitis media: the link with adenoids. Int J Immunopathol Pharmacol 2010;23(Suppl 1):38-40.

2. Izu SC, I tamoto CH, Pradella-Hallinan M, Pizarro GU, Tufik S, Pignatari S, et al. Obstructive sleep apnea syndrome (OSAS) in mouth breathing children. B raz J Otorhinolaryngol 2010;76:552556.

3. Waitrak BJ, Woolley AL. Pharyngitis and adenotonsilar disease. Em: Cummings CW, Flint PW, editors. Cummings 
otolaryngology head and neck surgery. 4th ed. Philadelphia: Elsevier M osby; 2005.p.4135-4139.

4. A lexopoulos EI, Kaditis A G, Kalampouka E, Kostadima E, A ngelopoulos NV, Mikraki V, et al. Nasal corticosteroids for children with snoring. Pediatr Pulmonol 2004;38:161-167.

5. SilvaV C, L eiteA J. Quality of lifein children with sleep-disordered breathing: evaluation by OSA-18. Braz J Otorhinolaryngol 2006;72:747-756.

6. Nave H, G ebertA, Pabst R. M orphology and immunology of the human palatine tonsil. A nat Embryol (B erl) 2001;204:367-373.

7. M ira J GS, Pasinato RC, M ocell in M, Ferreira N eto O, Capasso $R$, Catam GSA, et al. Efeitos da adenoamigdalectomia no sistema imune humoral. Rev Bras Otorhinolaryngol 1998; 64:437-440.

8. Faramarzi A, Shamseddin A, G haderi A . IgM , IgG serum levels and lymphocytes count before and after adenotonsillectomy. I ran J Immunol 2006;3:187-191.
9. Chadha NK , Zhang L, M endoza-Sassi RA, César J A. U sing nasal steroids to treat nasal obstruction caused by adenoid hypertrophy: does it work? O tolaryngol Head N eck Surg 2009;140:139-147.

10. Berlucchi M, Valetti L, Parrinello G, Nicolai P. Long-term follow-up of children undergoing topical intranasal steroid therapy for adenoidal hypertrophy. Int] Pediatr Otorhinolaryngol 2008;72:1171-1175.

11. Rezende RM, Silveira F, B arbosa A P. Objective reduction in adenoid tissue after mometasone furoate treatment. Int J Pediatr Otorhinolaryngol 2012;76:829-831.

12. Brouillette RT, M anoukian J , Ducharme FM, et al. Efficacy of fluticasone nasal spray for pediatric obstructive sleep apnea. J Pediatr 2001;138:838-840.

13. van den A kkerEH, Sanders EA, van Staaij BK, Rijkers GT, R overs $M M$, Hoes AW, et al. L ong-term effects of pediatric adenotonsillectomy on serum immunoglobulin levels: results of a randomized controlled trial. A nn A llergy A sthma I mmunol 2006;97:251-256. 